\title{
Technical trading and cryptocurrencies
}

\author{
Robert Hudson ${ }^{1} \cdot$ Andrew Urquhart ${ }^{2}$
}

Published online: 30 August 2019

(c) The Author(s) 2019

\begin{abstract}
This paper carries out a comprehensive examination of technical trading rules in cryptocurrency markets, using data from two Bitcoin markets and three other popular cryptocurrencies. We employ almost 15,000 technical trading rules from the main five classes of technical trading rules and find significant predictability and profitability for each class of technical trading rule in each cryptocurrency. We find that the breakeven transaction costs are substantially higher than those typically found in cryptocurrency markets. To safeguard against datasnooping, we implement a number of multiple hypothesis procedures which confirms our findings that technical trading rules do offer significant predictive power and profitability to investors. We also show that the technical trading rules offer substantially higher risk-adjusted returns than the simple buy-and-hold strategy, showing protection against lengthy and severe drawdowns associated with cryptocurrency markets. However there is no predictability for Bitcoin in the out-of-sample period, although predictability remains in other cryptocurrency markets.
\end{abstract}

Keywords Technical analysis · Cryptocurrencies · Bitcoin · Data-snooping · Market efficiency

\section{Introduction}

Cryptocurrencies are a new type of financial instrument that have received a great amount of interest both from the media and investors in the recent past. The first cryptocurrency created was Bitcoin, proposed by Nakamoto (2008), as a peer-to-peer electronic cash system which allows online payments to be sent directly from one party to another without going through a financial institutions. Therefore unlike most other financial instruments, Bitcoin has no association with any authority and has no physical representation. The value of Bitcoin is not based on any tangible asset or any country's economy and instead is based upon the security of an algorithm which traces all transactions. The potential use of Bitcoin as a medium of

\footnotetext{
$凶$ Andrew Urquhart

a.j.urquhart@icmacentre.ac.uk

Robert Hudson

robert.hudson@hull.ac.uk

1 Hull University Business School, University of Hull, Hull, UK

2 ICMA Centre, Henley Business School, University of Reading, Reading, UK
} 
exchange is attractive due to its low transaction costs, its peer-to-peer, global and governmentfree design. However users may be concerned by the lack of confidence in the system as well as the lack of acceptability of Bitcoin to make transactions. As discussed above, Bitcoin was conceived as a new type of currency rather than an investment asset. However, Fig. 1 shows the price of Bitcoin and other cryptocurrencies has increased substantially since 2010 with Baur et al. (2018) showing that Bitcoin accounts are mainly used as a speculative investment rather than an alternative currency or medium of exchange, which is supported by Corbet et al. (2018). The investment suitability of Bitcoin has been hotly debated. Charlie Munger, the billionaire investor and partner of Warren Buffet, for example, stated "I regard the bitcoin craze as totally asinine" (Financial Times, 2018). ${ }^{1}$ Much of the criticism of Bitcoin has been on the grounds of its lack of intrinsic value. Nonetheless, the academic literature examining the price dynamics of Bitcoin and other cryptocurrencies is growing, with a number of papers studying bubbles in cryptocurrency markets (Cheah and Fry 2015; Fry and Cheah 2016; Corbet et al. 2018), the market efficiency of Bitcoin markets (Urquhart 2016; Nadarajah and Chu 2017; Bariviera 2017; Tiwari et al. 2018), the hedging properties of Bitcoin (Brière et al. 2015; Dyhrberg 2016a, b; Bouri et al. 2017; Urquhart and Zhang 2019), the price discovery within Bitcoin exchanges (Brandvold et al. 2015; Brauneis and Mestel 2018) as well as price clustering Urquhart (2017). Nevertheless, the finance literature concerning Bitcoin and other cryptocurrencies is limited since they are fairly new financial assets.

An area that has received increasing attention in the cryptocurrency literature is the benefits of investing in cryptocurrencies. Kajtazi and Moro (2019) explore the effects of adding Bitcoin to an optimal portfolio (naïve, long-only, semi-constrained with and without Bitcoin shorting) by relying on the mean-CVaR approach for US, European and Chinese portfolio assets. They show that Bitcoin improves the returns of the portfolio, mostly from increased returns rather than lower volatility, and that Bitcoin has a role in portfolio diversification. Recently, Platanakis and Urquhart (2019) examine the out-of-sample benefit of including Bitcoin in eight popular asset allocation strategies in portfolios of stocks and bonds. They find that the inclusion of Bitcoin generates substantially higher risk-adjusted returns, where the results are robust to a different structure of estimation windows, the incorporation of transaction costs, the inclusion of a commodity portfolio, an alternative index for Bitcoin as well as two additional portfolio optimization techniques including higher moments with (and without) variance-based constraints.In respect of the optimisation of cryptocurrency portfolios Platanakis et al. (2018) examine the performance of nave (1/N) and optimal (Markowitz) diversification in a portfolio of four popular cryptocurrencies. They show there is very little to differentiate between nave diversification and optimal diversification. ${ }^{2}$

We complement the growing literature on cryptocurrencies by performing the first comprehensive study of technical trading in cryptocurrency markets in order to assess whether technical trading rules offer predictive power and profitability in various cryptocurrency markets. Technical trading is of particular interest in cryptocurrency markets for a number of reasons. The trading approach has substantial documented success in the conventional currency markets and to, some extent, in many asset markets. ${ }^{3}$ Since cryptocurrency markets have tended to follow strong trends since their inception, we have some prior that technical trading rules may be beneficial in cryptocurrency markets. In addition, the relative lack of information relevant for performing fundamental analysis on cryptocurrencies may elevate

\footnotetext{
${ }_{1}^{1}$ Financial Times (2018) https://www.ft.com/content/c8a47b42-11d4-11e8-8cb6-b9ccc4c4dbbb.

${ }^{2}$ For an up-to-date review of the literature on cryptocurrencies, see Corbet et al. (2019).

${ }^{3}$ For instance, Brock et al. (1992), Sullivan et al. (1999), Shynkevich (2012), Neely et al. (2014), Shynkevich (2016) amongst others explained below.
} 


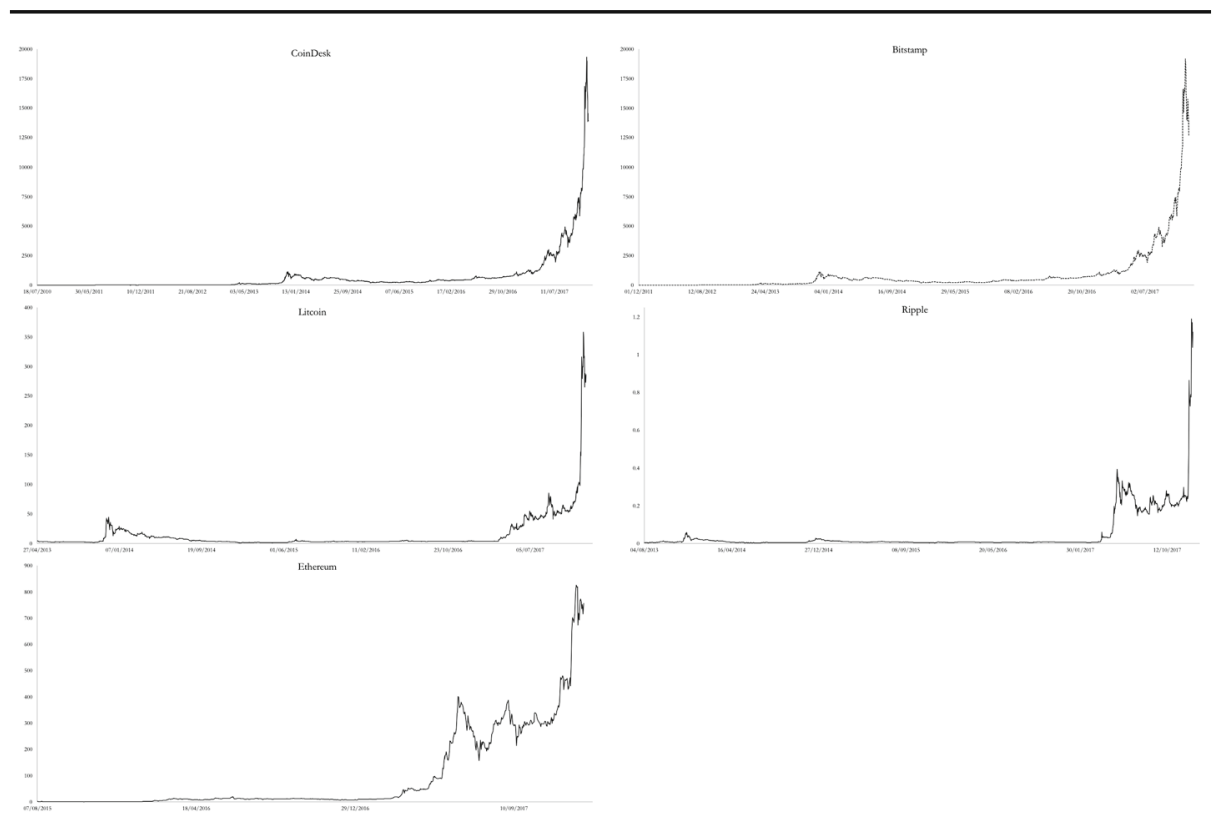

Fig. 1 Time-series graphs of the prices of the prices of the cryptocurrencies employed in this study

the relative importance of technical approaches. This is because cryptocurrencies have no fundamentals to examine and arguably have no intrinsic value. Therefore investors cannot study, for example, the balance sheet or dividend forecasts to forecast future prices and therefore have to rely on past price behaviour as a signal of future behaviour, which is the fundamental concept behind technical trading.

The only paper to our knowledge that examines technical trading in cryptocurrency markets is Detzel et al. (2018), who show that the 5- to 100-day moving average rule, both in- and out-of-sample, offers predictive power to investors. They also show that trading strategies based on these rules generate substantial alphas, utility and Sharpe ratios while significantly reducing the severity of drawdowns relative to a buy-and-hold position in Bitcoin. However this paper examines just one type technical trading rule while there are many different types of technical trading rules, with many different parameterizations. Investors have been shown to use a number of different technical trading rules and therefore we further the literature by studying a range of the most popular technical trading rules. Further although the Bitcoin market is the largest, other cryptocurrency markets have been gaining attention that should not be ignored since the growth of interest in the non-Bitcoin cryptocurrencies have grown exponentially over the last few years.

We study daily data from four different cryptocurrencies, namely Bitcoin, Litecoin, Ethereum and Ripple, for the longest data period available for each individual cryptocurrency. We consider a range of five different classes of technical trading rules (similar to Hsu et al. 2016), in which we examine a number of different parameters. As noted by Shynkevich (2012), choosing too few rules can cause biases in statistical inference due to data mining. On the other hand, using too many irrelevant rules can reduce the test power. We therefore look for a balance and select a fairly large variety of reasonable parameters within the five most popular families for a combined number of 14,919 trading rules. We employ a range of performance metrics to assess the returns from technical trading, including a number of 
risk-adjusted measures as well as the breakeven costs. Since we examine a number of different rules, we face the real possibility of data-snooping bias where the large number of hypotheses tested means that the likelihood of a rejecting the null hypothesis of each trading rule (making a Type I error) is quite high. To safeguard against this issue, we first calculate the individual bootstrapped $p$-values of each technical trading by comparing the actual $p$-value to 1000 stationary bootstrapped $p$-values following the method of Sullivan et al. (1999). We then use these individual bootstrapped $p$-values and adopt a number of approaches to control for multiple hypothesis testing, namely the family-wise error rate $(F W E R)$ and the false discovery rate $(F D R) .{ }^{4}$ Our results show that technical trading rules do offer predictive power in cryptocurrency markets where the average annualized return for each family of technical trading rule is statistically significant at the 5\% level for each cryptocurrency. Our results are robust to risk-adjusted measures and the breakeven transaction costs of the majority of rules examined are substantially higher than those found in cryptocurrency markets. After accounting for data-snooping via various multiple hypothesis testing procedures, a large proportion of technical trading rules still report significant returns indicating the predictive power and profitability of technical trading in cryptocurrency markets. More importantly however, we show that implementing technical trading rules significantly reduces the potential drawdowns faced by the buy-and-hold and therefore protects investors from the lengthy and severe drawdowns associated with cryptocurrency markets. Finally, we show that Bitcoin does not offer any positive returns in the out-of-sample period, but the other cryptocurrencies do offer positive returns and relatively highly Sharpe and Sortino ratios.

The success of technical trading rules to generate consistent profits has been an ongoing debate in the academic literature. Practitioners have been found to use technical analysis extensively, with Smith et al. (2016) showing that $21.6 \%$ of live hedge funds use technical analysis while Menkhoff (2007) reports that technical analysis is widespread in the foreign exchange market. Yet, the academic literature has heavily scrutinized the performance of technical analysis since it provides evidence against one of the most respected theories in finance, the efficient market hypothesis. Weak form market efficiency states that all available price information must be reflected in security prices and therefore the use of technical analysis must be futile. Since the foreign exchange market is where technical analysis is particularly widely used, studies have been plentiful and long indicated profit opportunities (for instance Poole 1967; Sweeney 1986; Levich and Thomas 1993; Neely et al. 1997; Gencay 1999; Qi and Wu 2006). This literature shows that simple technical trading rules on dollar exchange rates provided 15 years of positive, risk-adjusted returns during the 1970s and 1980s before those returns were extinguished (Olson 2004). In a comprehensive study, Hsu et al. (2016) study a large-scale study of technical trading rules in the foreign exchange market over 45 years in 30 developed and developing markets and find some evidence of substantial predictability and excess profitability in both developed and developing markets. Zarrabi et al. (2017) show that from 1994 to 2014, technical trading rules are profitable in six currencies quoted in U.S. dollars, however they are not consistently profitable.

The foreign exchange market is not the only market to report significant results from employing technical trading rules. In equity markets, Brock et al. (1992) show that technical trading provides significant predictability over 90 years for the Dow Jones Industrial Average, while Sullivan et al. (1999) and White (2000) show that the findings of Brock et al. (1992) are not due to data-snooping. Many other papers have also reported significant results for technical trading in equity markets, such as Shynkevich (2012), Han et al. (2013) and Neely et al. (2014). There is also evidence of significant results from technical trading in commodity

${ }^{4}$ See Harvey and Liu (2014) for more details on the issue of multiple hypothesis testing. 
futures markets (Miffre and Rallis 2007; Szakmary et al. 2010; Narayan et al. 2015; Han et al. 2016), commodity spot markets (Batten et al. 2018; Psaradellis et al. 2019), bond markets (Shynkevich 2016) and commodity ETFs (Hudson et al. 2017). Despite these findings, there is no clear consensus on the predictability of technical trading rules in the literature, with many papers indicating that technical trading rules do not offer any predictive power, especially once transaction costs have been accounted for (for instance Bessembinder and Chan 1998; Allen and Karjalainen 1999; Marshall et al. 2008a, b; Bajgrowicz and Scaillet 2012; Yamamoto 2012; Urquhart et al. 2015 ; Batten et al. 2018).

Therefore this paper offers a number of important contributions to the literature. Firstly, this is the first paper to provide a large-scale study of the performance of technical trading rules in cryptocurrencies. Although previous research has focused on a wide range of other financial assets only one paper (Detzel et al.2018) focuses on cryptocurrencies and that paper only investigates the Bitcoin cryptocurrency while we provide a comprehensive investigation of a number of major cryptocurrencies. Secondly, we employ a wide-range of technical trading rules to provide a thorough investigation of the performance of technical rules in cryptocurrency markets. We also employ risk-adjusted performance measures as well as compute breakeven transaction costs. Third, to safeguard against data-snooping we employ a number of multiple hypothesis tests. Finally, we study the in-sample and out-of-sample performance of technical trading rules in cryptocurrencies to safeguard against any datamining issues.

The rest of the paper is organized as follows. Section 2 discusses the dataset used in this study and provides descriptive statistics, while Sect. 3 describes the various technical trading rules that we implement. Section 4 outlines the various performance metrics considered in order to assess the performance of our rules while Sect. 5 sets out the data-snooping measures employed in this study. Section 6 reports the empirical results while Sect. 7 provides a summary and the conclusion.

\section{Data}

We employ daily Bitcoin prices from two providers for robustness purposes, namely CoinDesk and Bitstamp. CoinDesk represents an average of Bitcoin prices across leading global exchanges that meet criteria specified by CoinDesk. These criteria include that the exchange must serve an international customer base, must provide a bid-offer spread for an immediate sale (offer) and an immediate purchase (bid), while also the minimum trade size must be less than $\$ 1500$ USD. Also, the daily trading volume must meet minimum acceptable levels as determined by CoinDesk while the exchange must represent at least $5 \%$ of the total 30 -day cumulative volume for all of the exchanges included in the CoinDesk price. We also study an actual exchange Bitcoin price, Bitstamp, which is one of the first, most popular and liquid Bitcoin exchanges (Brandvold et al. 2015), is based in the UK and is considered to be a rather safe exchange by market participants around the world (Bouri et al. 2017). For the other cryptocurrencies, we obtain daily data from www.CoinMarketCap.com for Litecoin, Ethereum and Ripple, which are the largest and most liquid cryptocurrencies in the market. ${ }^{5}$ We study each cryptocurrency for the maximum period possible to ensure our results provide a complete picture of the performance of technical analysis in these cryptocurrencies. Specially, we study CoinDesk's Bitcoin price from 18th July 2010, Bitstamp from 1st December 2012,

5 www.CoinMarketCap.com has been employed previously in the literature to examine cryptocurrencies other than Bitcoin, such as Ciaian et al. (2018). 
Table 1 Descriptive statistics of the returns of Bitstamp, CoinDesk, Ethereum, Ripple and Litecoin from the start of available data to the 31st December 2017

\begin{tabular}{llllllll}
\hline Cryptocurrency & Mean & SD & Max & Min & Skewness & Kurtosis & Obs \\
\hline CoinDesk & 0.0061 & 0.0592 & 0.5289 & -0.3883 & 0.8328 & 12.1985 & 2735 \\
Bitstamp & 0.0049 & 0.0481 & 0.4014 & -0.4852 & 0.0693 & 14.2082 & 2217 \\
Litecoin & 0.0049 & 0.0769 & 1.2910 & -0.4019 & 5.0661 & 67.6347 & 1710 \\
Ripple & 0.0070 & 0.0927 & 1.7937 & -0.4600 & 6.3377 & 97.9744 & 1624 \\
Ethereum & 0.0098 & 0.0799 & 0.5070 & -0.7292 & 0.2199 & 13.1442 & 877 \\
\hline
\end{tabular}

Litecoin from 28th April 2013, 28th April 2013, Ripple from 4th August 2013 and Ethereum from 7th August 2015. All prices end on 31st December 2017. All markets trade 24 hours a day 7 days a week and therefore we include all observations during the day including weekends and holidays and have a complete time-series. Figure 1 presents the time-series graph of each cryptocurrency over time for the sample periods chosen in this study and each show the dramatic rise in the price of each cryptocurrency and therefore also the large volatility. We employ simple returns such that:

$$
r_{t}=\left(\frac{P_{t}-P_{t-1}}{P_{t-1}}\right)
$$

where $P_{t}$ is the price of the cryptocurrency at time $t$ and $P_{t-1}$ is the price of the cryptocurrency at time $t-1 .^{6}$ Table 1 reports the descriptive statistics of the returns of the various cryptocurrencies where we find that the all cryptocurrencies have a positive mean return, with Ethereum the largest and Litecoin the smallest where Ethereum has a mean return double that of Bitstamp and Litecoin. Ripple is the most volatile cryptocurrency while Bitstamp is the least volatile. The maximum and minimum values for each cryptocurrency document the extreme returns that can be found with these cryptocurrencies. All cryptocurrencies are positively skewed, reflecting the general upward trend in cryptocurrency prices. ${ }^{7}$ All cryptocurrencies exhibit excess kurtosis indicating the leptokurtic nature of these returns. Litecoin and Ripple are the most heavily positively skewed and have the largest excess kurtosis of all cryptocurrencies. Therefore Table 1 reflects the nature of cryptocurrency markets and how they are quite different to traditional financial assets.

\section{Technical trading rules}

Taylor and Allen (1992) note that technical analysis can be separated into two distinct categories, namely the qualitative form and the quantitative form. The qualitative form is where charts are analyzed and attempts are made to identify patterns in the data while the quantitative form is the analysis of past charts through time-series analysis to construct trading signals. The main difference between the two types is that, given a certain trading rule, quantitative technical analysis is completely objective and every individual should come to the same conclusion while qualitative technical analysis is subjective and individuals may come to different conclusions from the same chart.

\footnotetext{
6 We use simple returns in order to assess the profitability of technical trading rules from the perspective of an investor, similar to Bajgrowicz and Scaillet (2012).

7 This is unlike the vast majority of traditional assets which generally have negative skewness.
} 
We follow Hsu et al. (2016) and study five classes of technical trading rules that are commonly used by traders and examined in the literature. The first class is moving average rules (which are the most examined class of technical trading rule in the literature) which attempt to ride trends and identify imminent breaks by examining moving averages, and are quite similar to the time-series momentum effect, first outlined by Moskowitz et al. (2012) with a correlation of returns between the two in excess of 0.80 (see Marshall et al. (2017) for a comparison between time-series momentum and moving average rules). The second class of rules we study are filter rules which attempt to follow trends by buying (selling) whenever the price has increased (decreased) by a given percentage. The third class are support-resistance trading rules, which create support or resistance bounds around the price which if they breach, indicates further movement in the same direction. The fourth class we study are oscillator trading rules, which attempt to identify overbought (oversold) assets and therefore anticipate the imminent market correction. The final class of rule studied is the channel breakout rules which identify time-varying support and resistance levels which, once breached, indicate further movement in the same direction.

The choice of parameters to employ in these rules is quite important since different parameters may generate quite contrasting returns. Therefore we examine a number of different variants of each class of trading rule, and therefore obtain a very large number of different possible rules. "Appendix" presents more details of the different variants of each of the class of rules as well as the various parameterizations employed in this study. In total, we study 14,919 technical trading rules.

\section{Performance metrics}

The return from the $j$ th technical trading in each cryptocurrency market is defined as;

$$
R_{j, t}=S_{j, t-1} r_{t}
$$

where $S_{j, t-1}$ denotes the position guided by $j$ th technical trading rule which is determined by historic prices tracking back from the closing spot rate of period $t-1 . S_{j, t-1}$ either takes the value of 1 where we go long in the cryptocurrency, -1 where we go short in the cryptocurrency or 0 where we are neutral. If we are neutral, we are out of the market and invested in cash. ${ }^{8}$

Any analysis of trading strategies needs to be concerned with transaction costs since if the returns from a trading strategy are not positive after taking account of transaction costs, the strategy is worthless to an investor. This is especially important since many papers have found that technical trading rules are profitable for investors but once transaction costs are accounted for, many rules are no longer profitable. Lintilhac and Tourin (2017) show that transaction costs on Bitcoin is around 50 basis points. However the actual transaction costs may be very different on different exchanges, and since we study four different cryptocurrencies, transaction costs across cryptocurrency markets may differ. Therefore we report the breakeven transaction costs in order to determine the magnitude of transaction costs that would make technical trading returns zero.

8 We invest in cash if we are not long or short in the cryptocurrency, meaning our results are based purely on the benefits of employing technical analysis on Bitcoin since we take no returns from the risk-free rate. 
The first performance metric we report is the daily mean return of the $j$ th technical trading rule which is defined as;

$$
\bar{R}_{j}=\frac{1}{n} \sum_{t=1}^{T} R_{j, t}
$$

where $\bar{R}_{j}$ is the mean return. This is the simplest performance measure and quantifies the average return from each trade of each technical trading rule. However, an issue with this measure is that it does not take into account the riskiness of the trading rule in terms of volatility of returns. Therefore we also calculate the Sharpe ratio which is the standard performance metric in the finance industry and measures units of average excess return per unit of risk as measures by the standard deviation of excess returns. The Sharpe ratio of the $j$ th technical trading rue is defined as;

$$
S R_{j}=\frac{\bar{R}_{j}-r_{f}}{\sigma_{j}}
$$

where $r_{f}$ is the daily US risk free rate which is calculated from the monthly U.S. risk-free obtained from the Kenneth French data library and $\sigma_{j}$ is the standard deviation of excess returns generated by the $j$ th trading rule. ${ }^{9}$ However an issue with the Sharpe ratio is that it includes downside volatility as well as upside volatility, with the same weighting. Investors may only be interested in the downside risk as the upside provides them with greater returns. Consequently we also calculate the Sortino ratio which of the $j$ th technical trading rue is defined as;

$$
S O_{j}=\frac{\bar{R}_{j}-r_{f}}{\sigma_{n, j}}
$$

where $\sigma_{n, j}$ is the standard deviation of negative excess returns generated by the $j$ th trading rule and again we annualized the Sortino ratio. Our final performance metric is the Calmar ratio, which is an important indicator for investment banks as well as the hedge fund industry (Psaradellis et al. 2019). ${ }^{10}$ The Calmar ratio calculates the average annual return of an investment per unit of maximum drawdown and is especially useful for practitioners who employ momentum strategies that can suffer considerable drawdowns. The Calmar ratio of the $j$ th technical trading rue is defined as;

$$
C R_{j}=\frac{\bar{R}_{j}}{M D D_{j}}
$$

where $M D D_{d, j}$ is the maximum drawdown of the excess returns generated by the $j$ th trading rule.

\section{Data-snooping}

Data snooping bias is a real issue whenever any trading strategy is implemented since examining just the mean excess return across rules is not sufficient. Searching among a range of competing trading rules implicitly involves increasing the number of hypotheses tested as

\footnotetext{
9 Kenneth French data sets can be found at http://mba.tuck.dartmouth.edu/pages/faculty/ken.french/ data_library.html.

10 The Calmar ratio was developed by Young (1991) and stands for "California Managed Account Reports".
} 
poorly performing rules are disregarded. The problem of multiple hypothesis testing arises from the fact that as we increase the number of hypotheses being tested, we also increase the likelihood of a rare event and therefore, the likelihood of incorrectly rejecting the null hypothesis of each trading rule (making at Type I error). In our case, after testing $n$ number of trading rules, a skeptic would argue that they would have been surprised if we had not found any that performed extremely well. ${ }^{11}$ This is demonstrated by Chordia et al. (2018) who create 2.1 million trading strategies and apply various multiple hypothesis testing techniques to show the outperformance under single hypothesis testing are likely false. The surviving strategies have no theoretical underpinnings and therefore $p$-hacking is a serious problem and after correcting for it, outperforming trading strategies are very rare.

We adopt two broad approaches to deal with multiple hypothesis testing, namely familywise error rate (FWER) and the false discovery rate (FDR).

\subsection{FWER}

The strictest multiple hypothesis test is to try and avoid any false rejections. This translates to controlling the FWER, which is defined as the probability of rejecting even one of the true null hypothesis and therefore the FWER measures the probability of even one false discovery. We implement two main FWER tests.

\subsubsection{Bonferroni method}

The Bonferroni method is a single-step procedure since all $p$-values are compared to a single critical value. This critical $p$-value is $\alpha / M$, where $\alpha$ is the critical value chosen and $M$ is number of rules examined. For a large number of rules, this adjustment leads to an extremely small critical $p$-value which makes it very conservative and leads to a loss of power. The lack of power is due to the fact that is implicitly treats all test statistics as independent and therefore ignores cross-correlation that is bound to be present in the technical trading rules employed in this study.

\subsubsection{Holm method}

The Holm method is a stepwise adjustment that that rejects the null hypothesis of no outperforming rules if $p_{i} \leq \alpha /(M-i+1)$ for $i=1, \ldots$ Compared to the Bonferroni method, the Holm method becomes less strict for large $p$-values. Thus the Holm method typically rejects more hypotheses and is more powerful than the Bonferroni method. However, it also does not take into account the dependence structure of the individual $p$-values and is very conservative.

\subsection{FDR}

Rather than controlling for the number of false rejections, we can control for the proportion of false rejections of the False Discovery Proportion (FDP). FDR measures and controls the expected FDP among all discoveries where a multiple hypothesis testing method is said to control FDR at level $\delta$ if $F D R \equiv E(F D P) \leq \delta$, where the level $\delta$ is user-defined.

11 Or as Economics Nobel Laureate Ronald Coase put it, "if you torture the data long enough, it'll confess to anything". 


\subsubsection{BH method}

One of the earliest FDR controlling methods is by Benjamini and Hochberg (1995) and is a stepwise procedure that, assuming all individual $p$-values are ordered from smallest to largest and defining:

$$
j^{*}=\max \left\{j: p \leq \frac{j \times \delta}{M}\right\}
$$

where we reject all hypothesis $H_{1}, H_{2}, \ldots, H_{j}$. This is a step-up method that starts with examining the least significant hypothesis and moves up to more significant test statistics.

\subsubsection{BY method}

Although Benjamini and Hochberg (1995) show that their method measures FDR if the $p$ values are mutually independent, Benjamini and Yekutieli (2001) show that a more general control of FDR under a more arbitrary dependence structure of $p$-values can be achieved by replacing the definition of $j^{*}$ with:

$$
j^{*}=\max \left\{j: p \leq \frac{j \times \delta}{M \times C_{M}}\right\}
$$

where the constant $C_{M}=\sum_{i=1}^{M} 1 / i \approx \log (M)+0.5$. However this method is less powerful than the $B H$ method and is still very conservative.

We employ these four multiple hypothesis testing procedures on the individual $p$-values of each trading rule. Consistent with Bajgrowicz and Scaillet (2012), to acquire individual $p$-values, we follow the re-sampling procedure of Sullivan et al. (1999). We employ the stationary bootstrap method of Politis and Romano (1994) to resample the returns of each strategy, where the corresponding test statistic for each bootstrap series of returns is calculated by comparing the original $p$-value with the bootstrapped $p$-values. ${ }^{12}$

\section{Empirical results}

\subsection{Initial results}

Table 2 reports the summary of the performance of the technical trading rules across all cryptocurrencies. We present the average result for all parameterizations of each technical trading rule where we show that on average, all five classes of technical trading rules generate significant annualized returns for all cryptocurrencies studied. For all cryptocurrencies and technical trading rules, the average return from a buy signal is positive and statistically significant while the average return from a sell signal is mostly negative indicating that the positive returns from technical trading in cryptocurrencies comes from the buy signals rather than the sell signals. Across all cryptocurrencies, the filter rule and the channel breakout rule performed best, generating the highest annualized returns which is as high as $16.45 \%$ for Ripple. The results reported in Table 2 only show the returns but the riskiness of the returns are very informative for investors. Therefore we report a number of metrics allowing for risk in Table 3 where we find that the all of them are greater than zero indicating that

12 The block length is equal to 0.1 and the number of bootstrap realizations is set to 1000 , consistent with previous studies. 


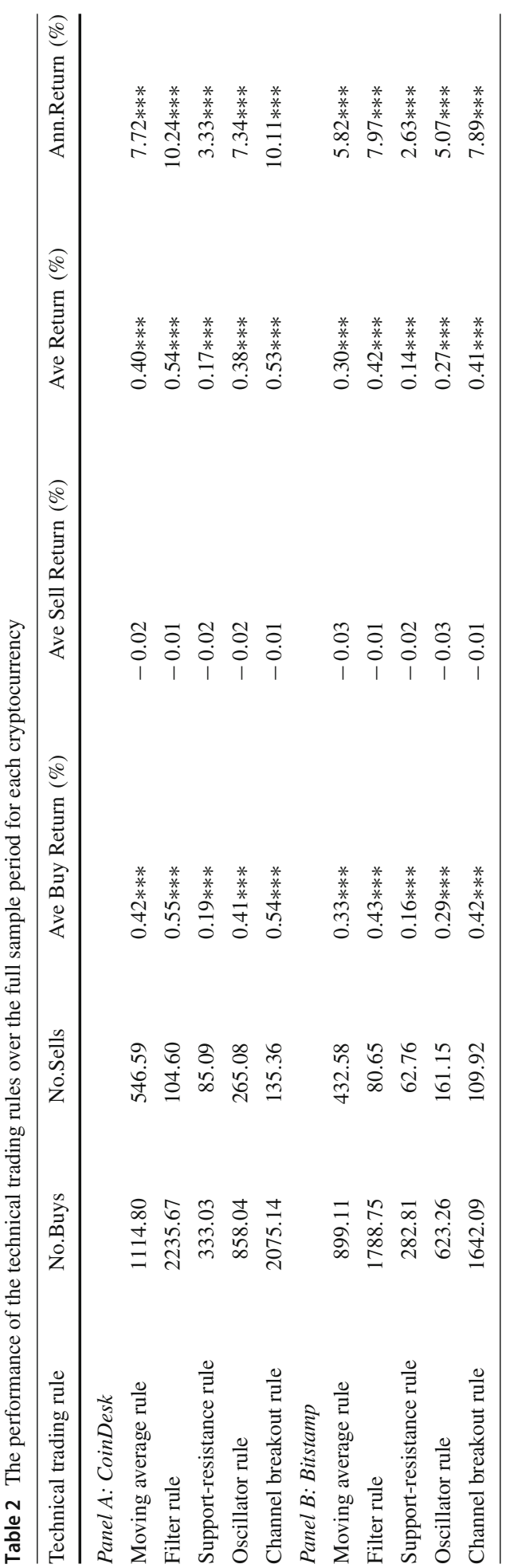




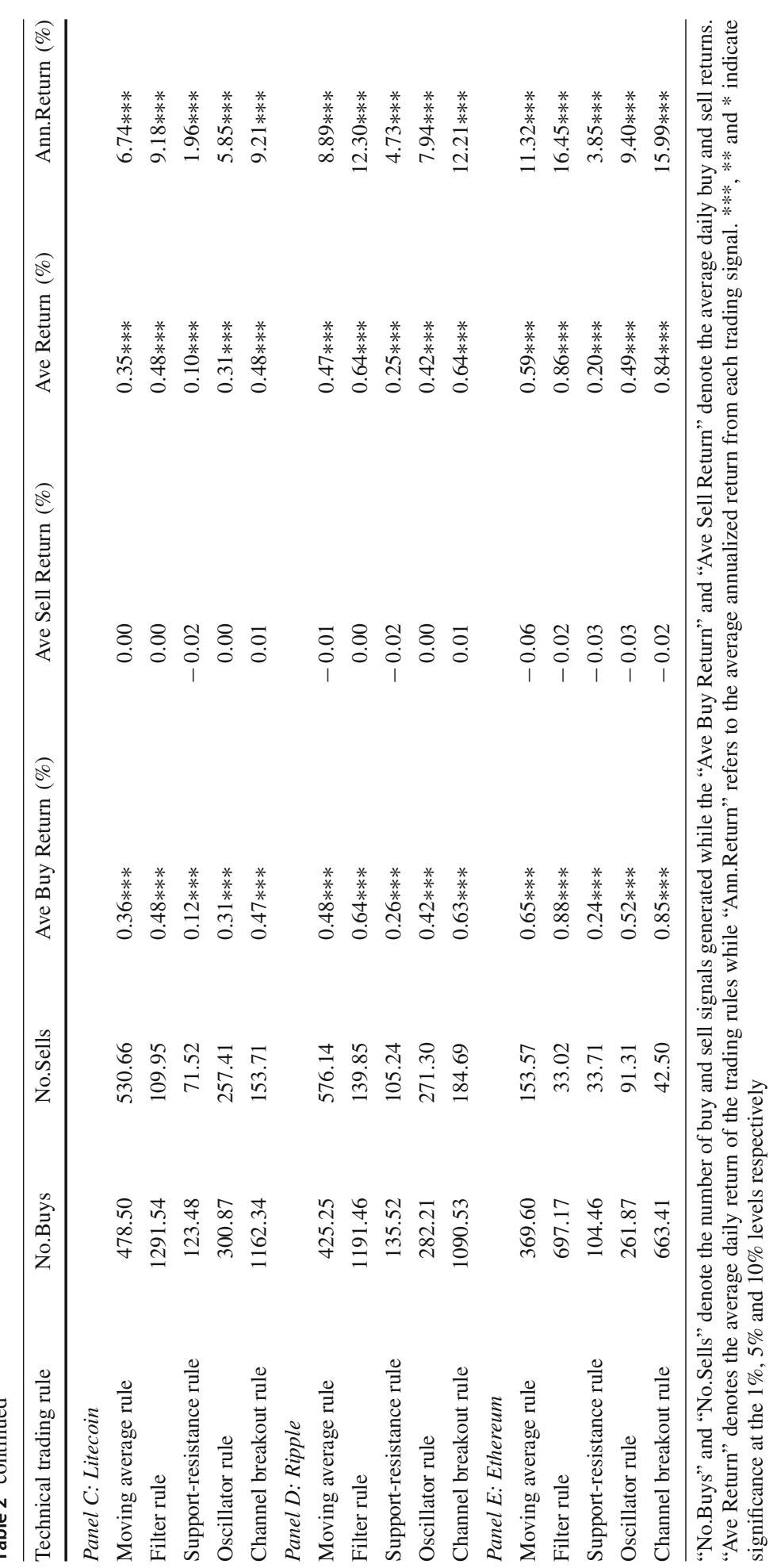




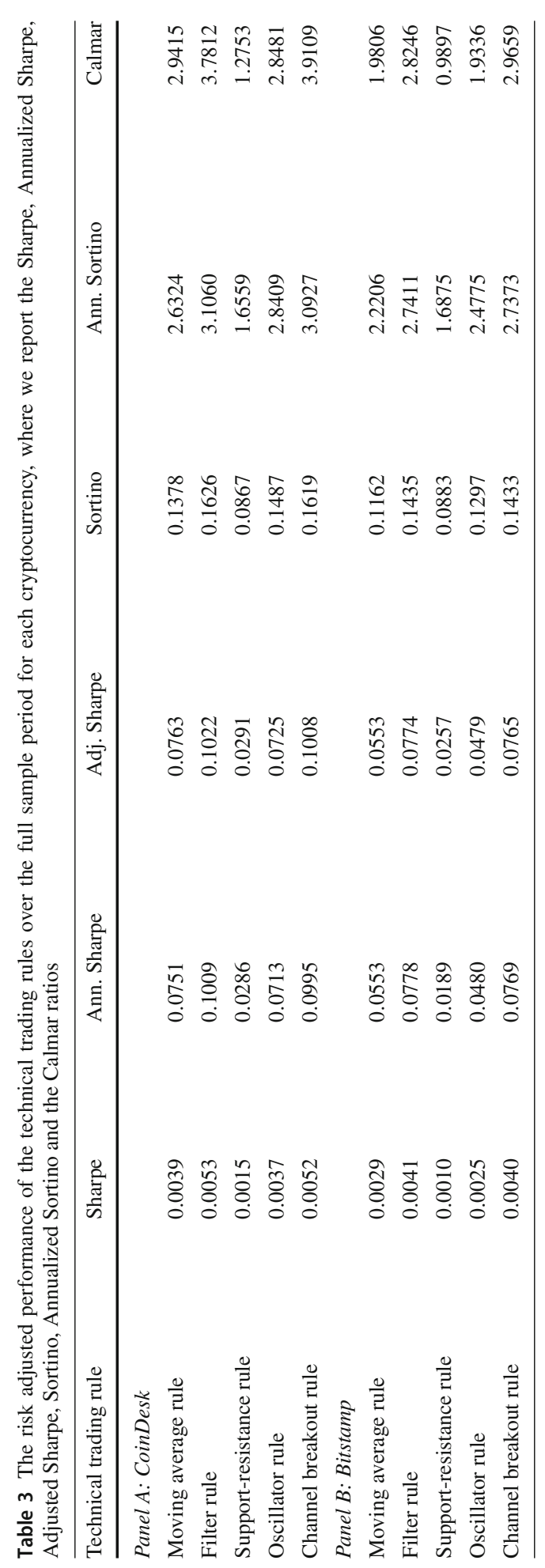




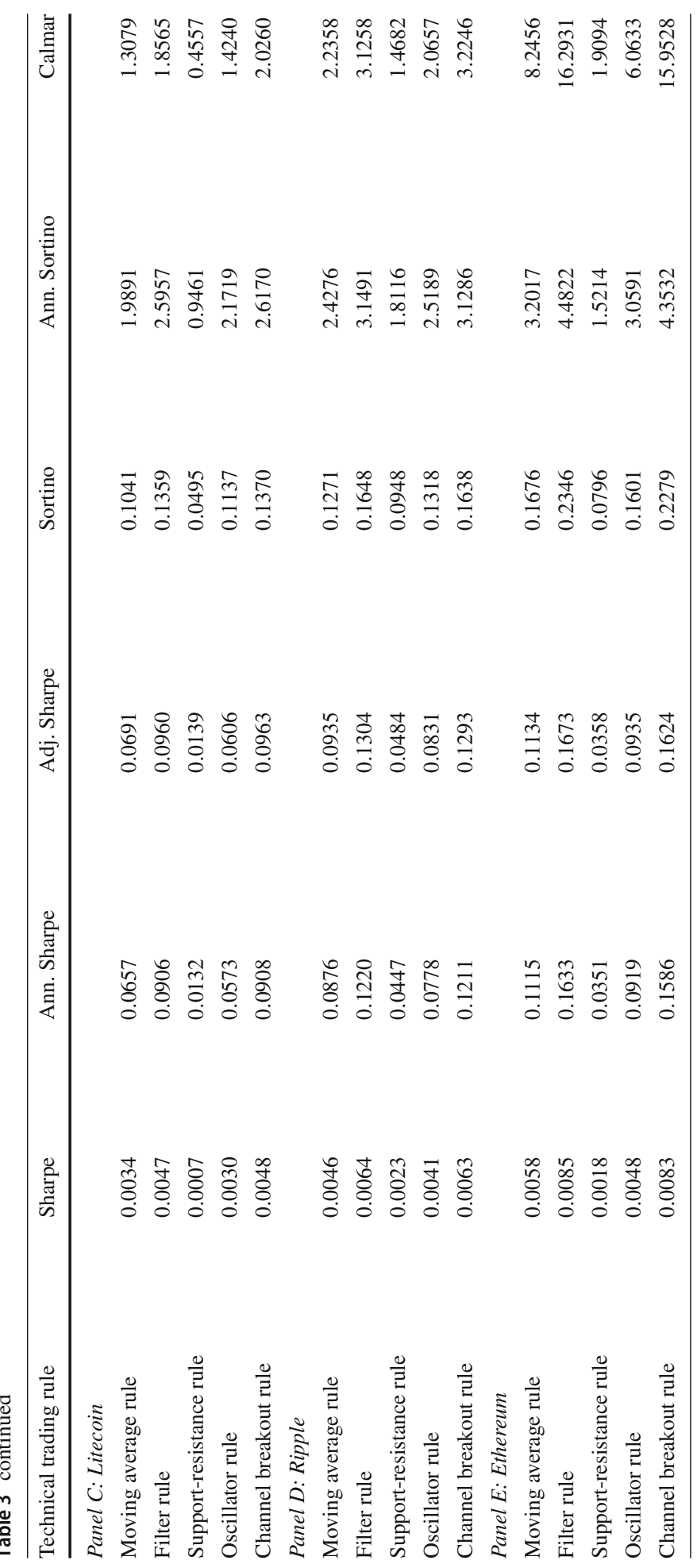


technical trading in cryptocurrencies outperforms the risk-free rate. We find that the Sortino ratio is much higher than the Sharpe and adjusted Sharpe ratios indicating that the riskiness from downside movements in returns is very limited. This is also supported by the Calmar ratio which, in each case, is quite large. Therefore our results show that on average, all classes of technical trading trading rules generate significant returns which provide favorable risk-adjusted metrics.

However it may be the case that not all of the different parameterizations of the technical trading rules will generate positive and significant returns. Therefore for each rule, we report the percentage of buy, sell and overall signals that produce positive and significant returns and present the results in Table 4 . Over $95 \%$ of technical trading rules generate positive buy returns in CoinDesk and Bitstamp, while over $90 \%$ of rules generate positive buy returns in Litecoin and Ripple. The vast majority of rules generate significant buy returns across all technical rule classes and across cryptocurrencies. The percentage of sell returns that generate positive returns is somewhat lower, ranging between only $1.97 \%$ for the Oscillator rule in CoinDesk, to $52.47 \%$ for the moving average rule in Litecoin. This suggests that only some of the parameterizations of each rule do generate positive returns from a sell signal. Also, we find that only a very small percentage of sell return signals do generate significant returns, with 15 out of the 20 technical trading rule classes across all cryptocurrencies not having any rules that generate a significant sell return after sell signals. The final two columns report the statistics for the overall returns and show that the vast majority of rules generate positive returns while over $50 \%$ of them generate significant returns at the $5 \%$ level, with the exception of the support-resistance rule for Litecoin, Ripple and Ethereum. Therefore the results in Table 4 show that although not all the technical trading rules generate positive and significant returns, quite a few still do generate significant returns for investors.

Given that some of the trading rules do perform well and some not so well, Table 5 reports the best and worst performing rules regarding annualized return, Sharpe and Sortino ratios. ${ }^{13}$ Clearly the worst performing rules generate annualized returns ranging from 2.68 to $-3.90 \%$, with negative Sharpe and Sortino ratios. However the best performing rules reported in the final three columns show substantial returns for investors. For example, the highest annualized return in CoinDesk is $13.42 \%$ while the highest for Ethereum is $22.15 \%$. The annualized Sharpe and Sortino ratios are also quite large indicating the risk-adjusted benefits of technical trading in cryptocurrencies. Therefore if investors can choose the best performing rules, then there are return making opportunities in applying technical trading rules to cryptocurrency markets.

\subsection{Transaction costs}

Up to this point, the analysis has assumed zero transaction costs but in practice these may be significant, even when trading cryptocurrencies. Indeed, a technical trading rule may predict cryptocurrency movements in the sense of generating significant returns but still not be profitable once the excess returns are adjusted for transaction costs (Timmermann and Granger 2004). Transaction costs in cryptocurrencies differ depending on the cryptocurrency traded and the exchange traded. Lintilhac and Tourin (2017) shows that the transaction fee per number of Bitcoins is $\$ 0.0025$ while the bid-ask spread is 0.005 as a fraction of the price is USD. However it is difficult to accurately estimate the transaction costs of CoinDesk since it is an average of the leading global exchanges of Bitcoin, while the transaction costs of the

13 All of the other performance metrics are not included to conserve space but are available from the corresponding author upon request. 


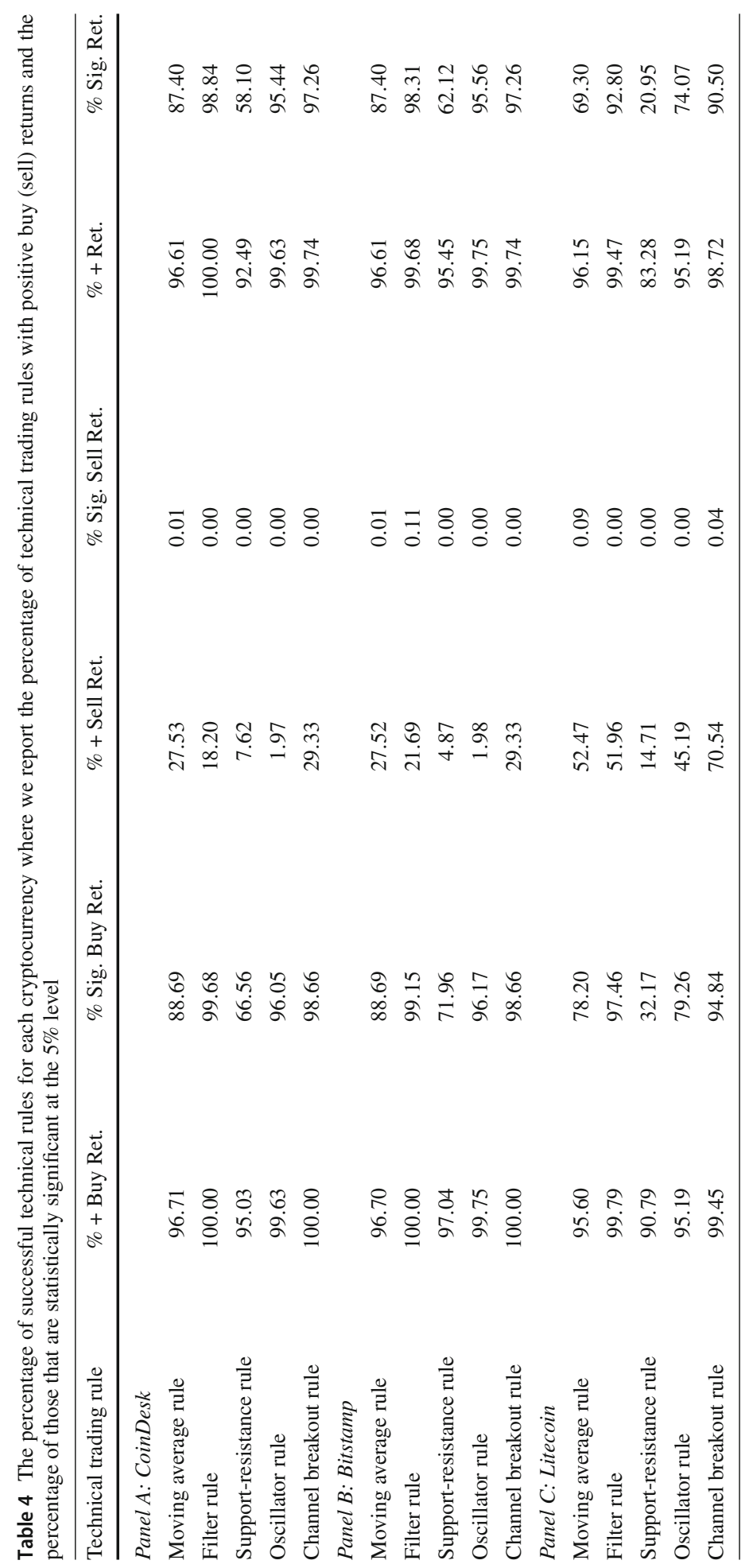




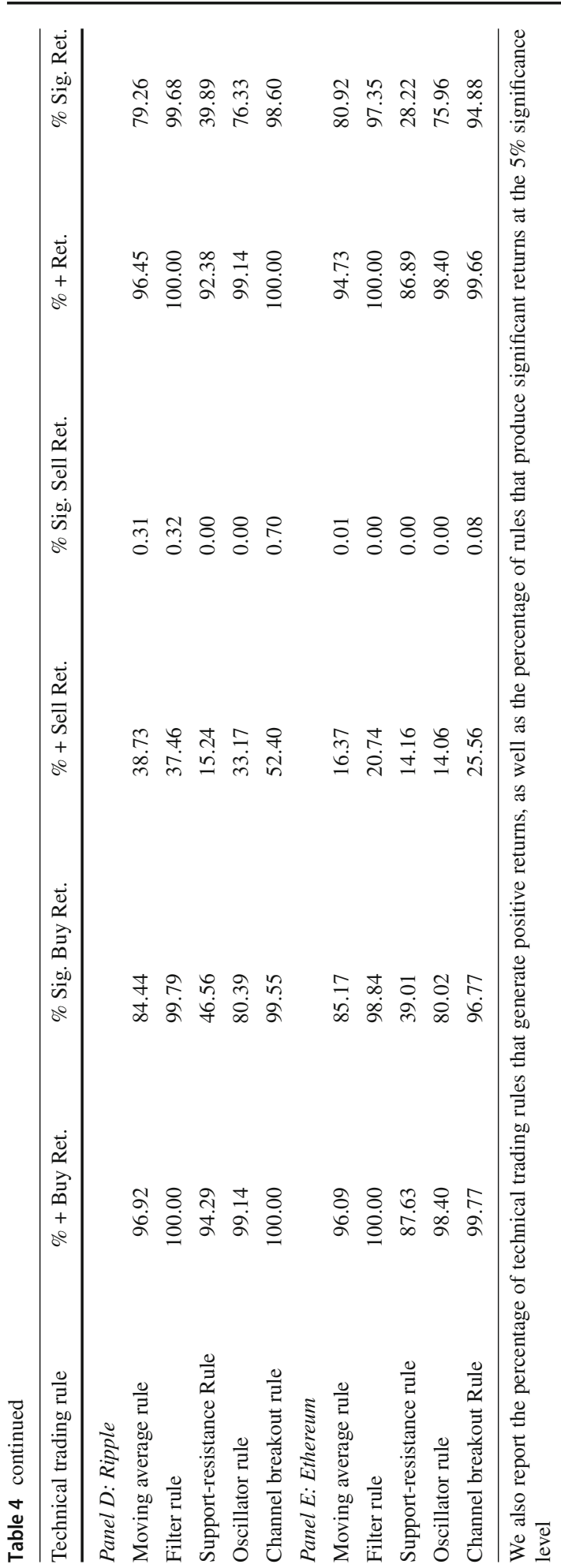




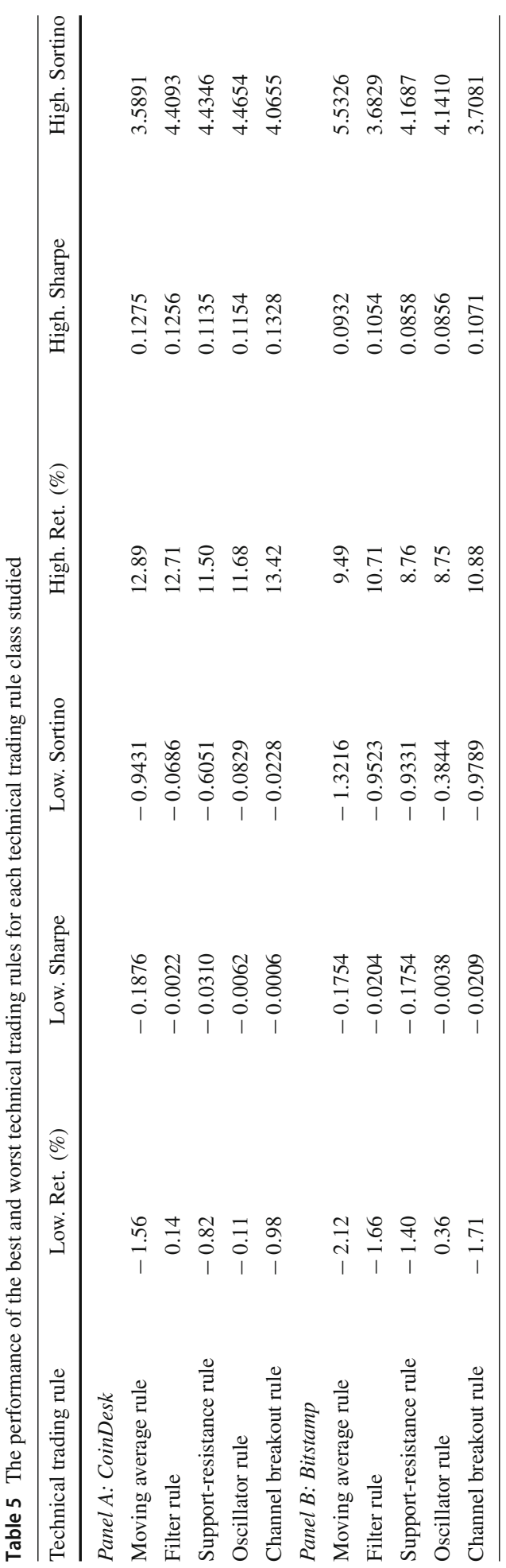

\section{照 Springer}




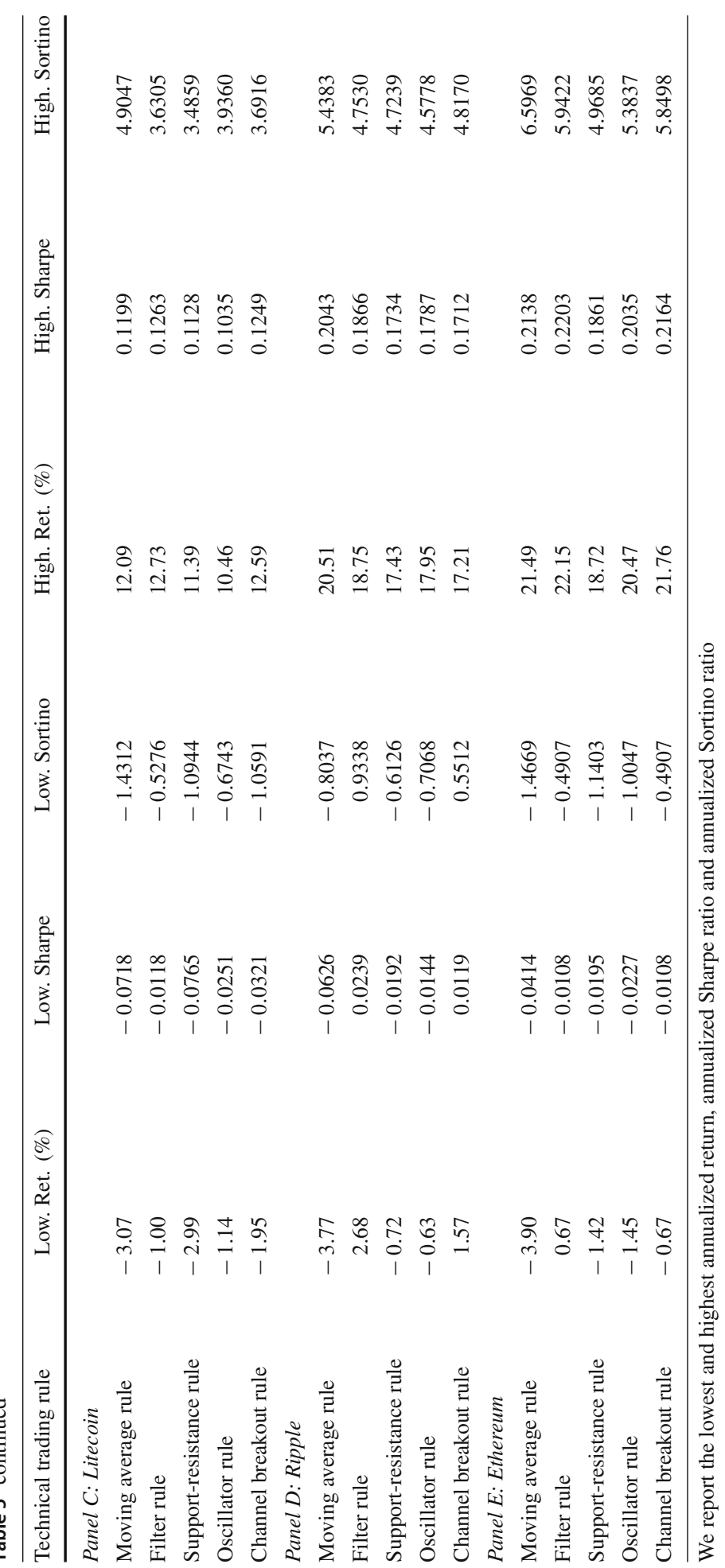


Table 6 The number of new trades generated by the technical trading rules for each cryptocurrency, the breakeven transaction costs in basis points and the percentage of rules that have greater breakeven transaction costs than 50 basis points

\begin{tabular}{|c|c|c|c|}
\hline Technical trading rule & No. trades & Breakeven TCs & $\%>50$ basis points \\
\hline \multicolumn{4}{|l|}{ Panel A: CoinDesk } \\
\hline Moving average rule & 182.98 & 54.86 & 32.04 \\
\hline Filter rule & 294.22 & 66.41 & 32.38 \\
\hline Support-resistance rule & 223.65 & 11.42 & 0.00 \\
\hline Oscillator rule & 210.89 & 44.60 & 36.06 \\
\hline Channel breakout Rule & 274.25 & 61.00 & 30.18 \\
\hline \multicolumn{4}{|l|}{ Panel B: Bitstamp } \\
\hline Moving average rule & 152.65 & 50.40 & 22.79 \\
\hline Filter rule & 238.43 & 57.51 & 28.15 \\
\hline Support-resistance rule & 184.06 & 11.89 & 0.95 \\
\hline Oscillator rule & 172.04 & 38.74 & 25.15 \\
\hline Channel breakout rule & 217.57 & 54.12 & 27.38 \\
\hline \multicolumn{4}{|l|}{ Panel C: Litecoin } \\
\hline Moving average rule & 137.39 & 35.44 & 25.81 \\
\hline Filter rule & 237.13 & 30.66 & 19.68 \\
\hline Support-resistance rule & 121.38 & 7.88 & 0.21 \\
\hline Oscillator rule & 134.73 & 38.42 & 24.44 \\
\hline Channel breakout rule & 214.77 & 30.42 & 17.97 \\
\hline \multicolumn{4}{|l|}{ Panel D: Ripple } \\
\hline Moving average rule & 128.55 & 36.01 & 26.25 \\
\hline Filter rule & 242.44 & 33.59 & 19.15 \\
\hline Support-resistance rule & 135.45 & 16.16 & 3.49 \\
\hline Oscillator rule & 127.45 & 52.14 & 34.04 \\
\hline Channel breakout rule & 219.22 & 33.06 & 18.37 \\
\hline \multicolumn{4}{|l|}{ Panel E: Ethereum } \\
\hline Moving average rule & 68.39 & 78.60 & 38.72 \\
\hline Filter rule & 99.83 & 147.56 & 49.52 \\
\hline Support-resistance rule & 76.66 & 9.89 & 0.11 \\
\hline Oscillator rule & 69.12 & 50.05 & 36.74 \\
\hline Channel breakout Rule & 93.53 & 144.03 & 47.35 \\
\hline
\end{tabular}

other cryptocurrencies may vary quite considerably over time. Therefore to avoid any bias in our results, we report in Table 6 the average breakeven transaction costs in basis points, along with the number of trades required. The number of trades are fairly constant within each cryptocurrency, although the filter rule does generate the highest number of trades across of cryptocurrencies. The breakeven transaction costs range 7.88 basis points for the supportresistance rule in Litecoin to 147.56 basis points for the filter rule in Ethereum. If we focus on CoinDesk and Bitstamp, the breakeven transaction costs are as high as 66.41 and 57.51 basis points respectively. Lintilhac and Tourin (2017) report that the transaction costs for Bitcoin is around 50 basis points and therefore also in Table 6 we report the percentage of technical trading rules that offer breakeven transaction costs greater than 50 basis points. Each class 
Table 7 This table reports the annualized return, Sharpe, Sortino and Calmar ratios for the buy-and-hold strategy in each cryptocurrency

\begin{tabular}{lllll}
\hline & Ann.Return & Ann.Sharpe & Ann.Sortino & Calmar \\
\hline CoinDesk & $0.1167(6.12 \%)$ & $0.1153(6.12 \%)$ & $3.1996(32.83 \%)$ & $4.22019(28.58 \%)$ \\
Bitstamp & $0.0942(4.96 \%)$ & $0.0925(4.86 \%)$ & $3.0163(32.14 \%)$ & $3.4522(32.72 \%)$ \\
Litecoin & $0.0928(11.49 \%)$ & $0.0918(41.30 \%)$ & $2.4167(51.26 \%)$ & $1.3750(57.64 \%)$ \\
Ripple & $0.1339(15.69 \%)$ & $0.1330(15.69 \%)$ & $3.1768(27.99 \%)$ & $2.7796(36.73 \%)$ \\
Ethereum & $0.1875(9.01 \%)$ & $0.1864(8.98 \%)$ & $3.9645(49.71 \%)$ & $11.0426(42.17 \%)$ \\
\hline
\end{tabular}

In brackets, we also report the percentage of technical trading rules that report returns higher than that of the buy-and-hold strategy

of technical trading rule in each cryptocurrency all generate a substantial percentage of rules that are greater than 50 basis points, with the exception of the support-resistance rule. For instance, $36.06 \%$ of oscillator rules generate breakeven transaction costs greater than 50 basis points for CoinDesk while $47.35 \%$ of channel breakout rules generate rules with breakeven transaction costs greater than 50 basis points for Ethereum. Very few support-resistance rules rules generate breakeven transaction costs greater than 50 basis points, indicating that this family of technical trading rules are not very successful for all four cryptocurrencies. Nevertheless, a large proportion of technical trading rules do generate breakeven transaction costs greater than 50 basis points indicating that performance of technical trading rules in cryptocurrencies are not wiped out by appropriate transaction costs.

\subsection{Comparison to the buy-and-hold strategy}

As shown in Fig. 1, cryptocurrencies have followed an upward trend over time, especially in the most recent time period. Therefore a buy-and-hold strategy may have been quite successful over the same sample period. Therefore in Table 7 we report the annualized return, annualized Sharpe ratio, annualized Sortino ratio and Calmar ratio for the buy-and-hold strategies for each cryptocurrency. In brackets, we also report the percentage of technical trading rules that generate risk-adjusted returns greater than the buy-and-hold strategy. For each cryptocurrency, we see that only a small proportion of rules generate annualized returns greater than the buy-and-hold strategy. However once we examine the risk-adjusted metrics, we find that substantially more technical trading rules offer returns greater than the buy-and-hold strategy, especially for the annualized Sortino and Calmar ratios, which both capture downside risk. This indicates that employing technical trading rules avoids the large, severe and lengthy drawdowns associated with cryptocurrencies and offers investors smoother returns than those that could have been gained from the simple buy-and-hold strategy. Therefore we show that if investors want to limit their risk exposure to cryptocurrency volatility, they could employ technical trading rules to smooth their future returns.

\subsection{MHT adjustment}

So far, we have shown that technical trading rules do offer predictive power to investors in five cryptocurrency markets. However as mentioned previously, there is an issue with datamining where searching among a range of competing rules is likely to result in at least a couple of rules generating significant results. In this paper, we examine in total 14,919 rules 
Table 8 The percentage of rules that remain significant at the 5\% level once various multiple hypothesis testing procedures are implemented

\begin{tabular}{llllll}
\hline & Bonferroni $(\%)$ & Holm $(\%)$ & BH $(\%)$ & BY (\%) & None $(\%)$ \\
\hline CoinDesk & 33.61 & 33.61 & 50.41 & 33.61 & 57.65 \\
Bitstamp & 27.11 & 27.13 & 46.28 & 28.56 & 58.64 \\
Litecoin & 23.26 & 23.26 & 31.84 & 23.26 & 53.23 \\
Ripple & 20.35 & 20.35 & 27.96 & 20.35 & 27.96 \\
Ethereum & 23.37 & 23.37 & 32.68 & 23.37 & 32.68 \\
\hline
\end{tabular}

"Bonferroni" refers to the Bonferroni adjustment, while "Holm" refers to the Holm adjustment. "BH" refers to the Benjamini and Hochberg (1995) adjustment while "BY" refers to the Benjamini and Yekutieli (2001) adjustment. "None" refers to the percentage of rules that are significant at the 5\% level after the stationary bootstrapping procedure of Politis and Romano (1994) is implemented

and therefore the probability of incorrectly rejecting the null hypothesis is quite high. To circumvent this issue, we adopt a number of different procedures where we first calculate each rule's individual bootstrapped $p$-value, which involves employing the stationary bootstrap of Politis and Romano (1994) to resample the returns of each strategy and the individual $p$-values are generated by comparing the original $p$-value with the bootstrapped $p$-values. Table 8 presents the percentage of individual bootstrapped $p$-value returns are significant at the 5\% level after the Bonferroni, Holm, BH, BY and no adjustment. The Bonferroni adjustment is the most restrictive of all the multiple hypothesis testing procedures where for each cryptocurrency, it suggests the lowest number of rules generate significant returns. However we can see that for each cryptocurrency, a large proportion of rules are significant even after accounting for multiple hypothesis testing. For instance, $33.61 \%$ of rules are still statistically significant after the Bonferroni and Hold adjustments for CoinDesk indicating that over a third of the rules generate significant returns. Although this ia a sharp decline coompared to the number of significant rules reported in Table 4, it clearly shows that technical rules are profitable in the cryptocurrency markets.

\subsection{Out-of-sample performance}

Another solution to address the data-snooping issue is to employ an out-of-sample analysis to examine if the best technical trading rules from the in-sample estimation performs well out of sample (Sullivan et al. 1999; Harvey et al. 2016). However one of the issues when examining the out-of-sample performance of any investment strategy is the choice of the in- and out-ofsample periods. This is especially important for technical trading rules since there is a trend in the literature for the performance of the rules to diminish over time (Menkhoff 2007). Further, McLean and Pontiff (2016) show that the performance for predictability variables substantially diminish after publication in the academic literature. Also, an out-of-sample estimation is very important with the respect of cryptocurrencies. Their volatility has been extremely high, which has put off a number of institutions and investors from including them in their portfolios. Further, there was a huge surge in the price of cryptocurrencies in the second half of 2017, but especially Bitcoin where the price briefly hit $\$ 20,000$. The first draft of this paper employed data up to 31st December 2017, but since then, there has been a huge drop in the value of cryptocurrencies, with the price hovering around $\$ 6500$ in early July 2018. therefore we can offer a pure out-of-sample analysis to determine whether technical 
Table 9 This table reports the out-of-sample results for the best rule in the in-sample period

\begin{tabular}{llccc}
\hline & Best rule & Ann.Return & Ann.Sharpe & Ann.Sortino \\
\hline CoinDesk & CB2: $25 / 0.05 / 0.025 / 5$ & -0.0010 & -0.0502 & -0.3470 \\
Bitstamp & CB2: $25 / 0.05 / 0.025 / 3$ & -0.0091 & -0.0641 & -0.0553 \\
Litecoin & CB2: $25 / 0.05 / 0.025 / 5$ & 0.0775 & 1.3553 & 2.1900 \\
Ripple & MA1: $2 / 0.001 / 1$ & 0.0546 & 0.7380 & 1.2162 \\
Ethereum & MA4: $2 / 25 / 0 / 1 / 5$ & 0.0631 & 1.1900 & 1.8500 \\
\hline
\end{tabular}

We report the best rule in the in-sample period for each cryptocurrency and then report the performance of that rule in the out-of-sample period. Specially, we report the annualized return, annualized Sharpe ratio and the annualized Sortino ratio. The coding for the best in-sample rules correspond to the description of the rules in the "Appendix"

analysis in cryptocurrencies does offer predictive power as well as examine how they have performed during the cryptocurrency bear market of the first half of 2018.

We provide a glimpse of the performance investors could have obtained over a pure out-of-sample period and during the downturn in the cryptocurrency markets. Specially, we examine the performance of the best performing rules up to 31st December 2017 and examine how they perform during the first 6 months of 2018, where the results are reported in Table 9. The channel breakout rule produces the best performing rules (all very similar in terms of parameters) for both Bitcoin prices, and Litecoin for the in-sample period, while moving average rules are the best performing in-sample technical trading rules for Ripple and Ethereum. In the out-of-sample periods, we find negative annualized returns, Sharpe ratios and Sortino ratios for both Bitcoin prices. However, the three other cryptocurrencies show positive out-of-sample returns, as well as positive Sharpe and Sortino ratios. This indicates that Bitcoin may not be profitable to trade using technical analysis in an out-of-sample setting, while the other cryptocurrencies we study, still generate substantial returns as well as quite large Sharpe and Sortino ratios in the out-of-sample period.

Bitcoin may be the least profitable cryptocurrency in the out-of-sample setting Bitcoin was the first cryptocurrency created as well as the most liquid and therefore attracts more attention from investors. The relatively large number of investors investors that are attracted to Bitcoin means that profitable trading strategies may be more difficult to find as the market becomes more efficient. This is also reflected in the academic literature where Corbet et al. (2019) show that the majority of academic papers on cryptocurrencies solely focus on Bitcoin. Also, the other cryptocurrencies we study are less liquid and therefore have attracted less attention from investors suggesting that they may offer more profit-making opportunities than Bitcoin.

\section{Conclusion}

Technical analysis has a long and rich history in the academic literature, with many papers reporting significant profitability in foreign exchange markets, stock markets, stocks and commodities. Although the reported profitability of technical trading has declined over time, there is still strong evidence that investors pay attention to technical trading rules and implement them as part of their investment strategies. We provide a comprehensive study on the benefit of employing a wide-range of technical trading rules in cryptocurrency markets. Therefore we provide a large-scale investigation of the profitability of technical trading rules across five of the most liquid and profitable cryptocurrencies by employing a large number of 
technical trading rules. Furthermore, we also employ a number of multiple hypothesis tests to safeguard against data-snooping bias.

We employ five families of technical trading rules (consistent with Hsu et al. 2016) in all five cryptocurrency markets and find that technical trading rules have strong predictive power. The annualized average return for each family of rule in each cryptocurrency market are all statistically significant at the 5\% level indicating the robustness of the results. The Sharpe, Sortino and Calmar ratios are also high, indicating the high risk-adjusted returns from implementing technical trading rules in the cryptocurrency sphere. We also report the breakeven transaction costs which generally show a value that is higher than commonly seen in cryptocurrencies indicating that the profits generated from technical trading are not wiped out by the inclusion of transaction costs. We also show that employing technical trading rules in cryptocurrencies generates much more favorable risk-adjusted measures than the simple buyand-hold strategy, and can protect investors from the large, severe and lengthly drawdowns associated with cryptocurrencies. Finally, we implement four popular adjustments of multiple hypothesis testing to safeguard against data-snooping bias and find that a large proportion of rules are still statistically significant, ranging from $20.35 \%$ of total rules to a high of $50.41 \%$. Finally, we show that technical trading rules cannot generate positive returns in the out-of-sample period for Bitcoin, but can for other cryptocurrencies. Therefore our results demonstrate that technical trading rules have significant predictive power in cryptocurrency markets even after accounting for multiple hypothesis testing, but Bitcoin does not offer any predictability in the out-of-sample period.

In this paper, we use both the Bitstamp Bitcoin price as well as the Coindesk Bitcoin price, which is the average across Bitcoin exchanges that meet a certain criteria specified by Coindesk. We do find technical trading rules performance is slightly better for Coindesk than for Bitstamp, although this difference is minimal. This difference could be due to the fact that Coindesk is the average across numerous exchanges and is intended to serve as a standard retail price reference for industry participants and accounting professionals. Therefore it is a hypothetical price across exchanges for Bitcoin and may offer more inefficiencies that the actual price of Bitcoin provided by Bitstamp.

Our paper fits into the literature supporting the implementation of technical trading rules where there are numerous studies suggesting that technical trading rules are profitable in financial markets. For instance, Hsu et al. (2016) shows that technical trading is successful in foreign exchange markets over the long run which is supported by the findings of Zarrabi et al. (2017), while Psaradellis et al. (2019) shows the success of technical trading rules in the oil market. We also add to the literature on the trading opportunities that are present in cryptocurrencies where Platanakis and Urquhart (2019) as well as Kajtazi and Moro (2019) show that the inclusion of Bitcoin can substantially improve the risk-adjusted returns of a stock-bond portfolio. Our findings support the findings of Detzel et al. (2018) who show that for Bitcoin trading strategies based on moving average ratios generate economically significant alphas and Sharpe ratio gains relative to a buy-and-hold strategy. While the Detzel et al. (2018) paper studies Bitcoin, we provide a wider study examining five of the largest and most liquid cryptocurrencies and therefore will be of interest to technical traders and cryptocurrency enthusiasts alike. We also investigate a wider range of technical trading rules than the moving average rules used in the Detzel et al. (2018) paper.

Open Access This article is distributed under the terms of the Creative Commons Attribution 4.0 International License (http://creativecommons.org/licenses/by/4.0/), which permits unrestricted use, distribution, and reproduction in any medium, provided you give appropriate credit to the original author(s) and the source, provide a link to the Creative Commons license, and indicate if changes were made. 


\section{Appendix}

This appendix reports further details on the technical trading rules employed in this study, as well as the parameters of each rule where we broadly follow the class of technical trading rules examined by Hsu et al. (2016)

\section{Moving Average Trading Rules}

Moving average trading rules are among the most widely used technical trading rules by traders (Taylor and Allen 1992) and they attempt to ride trends and identify imminent breaks in the trend or the emergence of new trends. For example, the most basic moving average rule is if the current price is larger (smaller) than the average of some number of previous prices, a buy (sell) signal is generated. However traders often replace the current price with a short-term moving average, or state the short-term moving average must be greater than the long-term moving average by a certain percentage, or persist for a certain number of days. We define a simple moving average as:

$$
M A_{t}(j)=\frac{1}{j} \sum_{i=0}^{j-1} s_{t-i}
$$

We study five different moving average rules, namely:

MA1: If the Bitcoin price moves up as least $x$ above $M A_{t}(q)$ and remains so for $d$ periods, go long until its price moves down at least $x$ percent below $M A_{t}(q)$ and remains so for $d$ periods, at which time go short. If the price moves down at least $x$ percent below $M A_{t}(q)$ and remains so for $d$ periods, go short until the price moves up at least $x$ above $M A_{t}(q)$ and remains so for $d$ periods, at which time we go long in the currency.

MA2: If the Bitcoin price moves up as least $x$ above $M A_{t}(q)$ and remains so for $d$ days, go long for $k$ periods and then neutralize the positions. If the price moves down at least $x$ percent below $M A_{t}(q)$ and remains of or $d$ days, go short for $k$ days and then neutralize the position.

MA3: If $M A_{t}(p)$ moves up at least $x$ percent above $M A_{t}(q)$ and remains so for $d$ periods, go long until $M A_{t}(p)$ moves down at least $x$ percent below $M A_{t}(q)$ and remains so for $d$ periods, at which time go short. If $M A_{t}(p)$ moves down at least $x$ percent below $M A_{t}(q)$ and remains so for $d$ periods, go short until $M A_{t}(p)$ moves up at least $x$ percent above $M A_{t}(q)$ and remains so for $d$ periods, at which time go long. $p$ is less than $q$.

MA4: If $M A_{t}(p)$ moves up at least $x$ percent above $M A_{t}(q)$ and remains so for $d$ periods, go long for $k$ periods, and then neutralize the position. If $M A_{t}(p)$ moves down at least $x$ percent below $M A_{t}(q)$ and remains so for $d$ periods, go short for $k$ periods and then neutralize the position. $p$ is less than $q$.

MA5: If the price moves up at least $x$ percent above any two of $M A_{t}(n), M A_{t}(p)$ and $M A_{t}(q)$ and remains so for $d$ periods, go long with one third of the risk budget. If the price moves up at least $x$ percent above all three of $M A_{t}(n), M A_{t}(p)$ and $M A_{t}(q)$ and remains so for $d$ periods, go long with all of the risk budget. If the price moves down at least $x$ percent below any two of $M A_{t}(n), M A_{t}(p)$ and $M A_{t}(q)$ and remains so for $d$ periods, go short with one third of the risk budget. If the price moves down at least $x$ percent below all three of $M A_{t}(n), M A_{t}(p)$ and $M A_{t}(q)$ and remains so for $d$ periods, go short with the whole risk budget. $n$ is less than $p$, which is less than $q$.

We consider a number of different parameter specifications, namely:

$q \in\{2,5,10,15,20,25,50,100,200\}, \# q=9$

$p \in\{2,5,10,15,20,25,50,100,200\}, \# p=9$

$n \in\{2,5,10,15,20,25,50,100,200\}, \# n=9$ 
$x \in\{0,0.01,0.05,0.1,0.5,1,5\}, \# x=7$

$d \in\{1,2,3,4,5\}, \# d=5$

$k \in\{3,5\}, \# k=2$

Therefore, we study the following number of parameterizations per rule:

$M A 1=\# q \times \# x \times \# d=9 \times 7 \times 5=315$

$M A 2=\# q \times \# x \times \# d \times \# k=9 \times 7 \times 5 \times 2=945$

$M A 3=\#(p-q) \times \# x \times \# d=36 \times 7 \times 5=1260$

$M A 4=\#(p-q) \times \# x \times \# d \times \# k=36 \times 7 \times 5 \times 2=2520$

$M A 5=\#(n-p-q) \times \# x \times \# d=84 \times 7 \times 5=2940$

Therefore the total $M A$ rules studied is 7980 .

2. Filter Trading Rules

The filter rule is one of the first and simplest technical trading rules and follows the basic premise that investors should buy an asset when its price has risen by more than a given percentage above its most recent low, or sell it when the price falls by more than a given percentage below its most recent high. We study three different filter rules, namely:

F1: If the Bitcoin price moves up at least $x$ percent above its most recent low and remain so for $d$ periods, go long. If the Bitcoin price moves down below its most recent high by at least $x$ percent and remains so for $d$ periods, go short. We define the most recent high (low) as the most recent closing price that is greater (less) than the $j$ previous prices, for a given value of $j$.

F2: If the Bitcoin price moves up at least $x$ percent above its most recent low and remains so for $d$ days, go long for $k$ periods and then neutralize the position. If the price moves down at least $x$ percent below its most recent high and remains so for $d$ periods, go short for $k$ periods and then neutralize the position.

We consider a number of different parameter specifications, namely:

$x \in\{0,0.01,0.05,0.1,0.5,1,5\}, \# x=7$

$d \in\{1,2,3,4,5\}, \# d=5$

$j \in\{2,5,10,15,20,25,50,100,200\}, \# j=9$

$k \in\{3,5\}, \# k=2$

Therefore, we study the following number of parameterizations per rule:

$F 1=\# x \times \# d \times \# j=7 \times 5 \times 9=315$

$F 2=\# x \times \# d \times \# k \times \# j=7 \times 5 \times 2 \times 9=648$

Therefore the total $F$ rules studied is 963 .

3. Support-Resistance Trading Rules

Support-resistance trading rules generate levels in which the price appears to find difficulty rising beyond (resistance) or difficulty in penetrating (support). The main idea is that a break of either the resistance or support levels will indicate a further movement in the same direction. Therefore they are quite similar to filter rules except a signal is generated when the price moves beyond a support or resistance level by a certain percentage, rather than beyond a recent high or low. We define a resistance level as the highest price of the $j$ previous prices and we define a support level as the lowest closing rates of $j$ previous closing and the two rules we examine are:

SR1: If the price moves up at least $x$ percent above the highest closing of the $j$ previous price and remains so for $d$ periods, go long. If the price moves down at least $x$ percent below the lowest closing of the previous $j$ prices and remains so for $d$ days, go short.

SR2: If the price moves up at least $x$ percent above the highest price of the $j$ previous prices and remains so for $d$ days, go long for $k$ days and then neutralize the position. If the price moves down at least $x$ percent below the lowest price of the $j$ previous closing rates and remains so for $d$ days, go short for $k$ days and then neutralize the position. 
We consider a number of different parameter specifications, namely:

$x \in\{0,0.01,0.05,0.1,0.5,1,5\}, \# x=7$

$d \in\{1,2,3,4,5\}, \# d=5$

$j \in\{2,5,10,15,20,25,50,100,200\}, \# j=9$

$k \in\{3,5\}, \# k=5$

Therefore, we study the following number of parameterizations per rule:

$S R 1=\# x \times \# d \times \# j=7 \times 5 \times 9=315$

$S R 2=\# x \times \# d \times \# j \times \# k=7 \times 5 \times 9 \times 2=630$

Therefore the total $S R$ rules studied is 945 .

4. Oscillator Trading Rules

A popular type of technical trading rule employed in the foreign exchange market is overbought/oversold indicator, which aims to indicate that the price movement in a particular direction has been too rapid and that a correction is imminent. One of the most popular oscillator rules is the relative strength indicator $(R S I)$ which is:

$$
R S I_{t}(h)=100\left[\frac{U_{t}(h)}{U_{t}(h)+D_{t}(h)}\right]
$$

where $U_{t}(h)$ denotes the cumulated up movement of prices over the previous $h$ periods, and $D_{t}(h)$ denotes the cumulative absolute downward movement over the previous $h$ periods. Therefore the $R S I$ indicator measures the strength of upward movements relative to downward movements and is normalized to lie between 0 and 100 . We examine two $R S I$ rules, namely:

O1: If $R S I_{t}(h)$ moves above $50+v$ for at least $d$ periods and then subsequently moves below $50+v$, go short. If $R S I_{t}(h)$ moves below $50-v$ for at least $d$ periods and then subsequently moves above $50-v$, go long.

O2: If $R S I_{t}(h)$ moves above $50+v$ for at least $d$ periods and then subsequently moves below $50+v$, go short for $k$ periods and then neutralize the position. If $R S I_{t}(h)$ moves below $50-v$ for at least $d$ periods and then subsequently moves above $50-v$, go long for $k$ periods and then neutralize the position.

We consider a number of different parameter specifications, namely:

$h \in\{2,5,10,15,20,25,50,100,200\}, \# h=9$

$v \in\{2,5,10,15,20,25\}, \# v=6$

$d \in\{1,2,3,4,5\}, \# d=4$

$k \in\{2,5\}, \# k=2$

Therefore, we study the following number of parameterizations per rule:

$O 1=\# h \times \# v \times \# d=9 \times 6 \times 5=270$

$O 2=\# h \times \# v \times \# d \times \# k=9 \times 6 \times 5 \times 2=540$

Therefore the total $O$ rules studied is 810 .

5. Channel Breakout Trading Rules

A channel breakout trading rule generates a channel around the price when the highest price over a previous given period is within a given range of the lowest level over the previous given period so that, there are time-varying support and resistance levels that appear to be drifting together within a certain range. A trading signal is therefore generated when the price penetrates beyond the upper or lower bond of the channel since the rule suggests that once a channel is breached, there will be sustained movement in the same direction. A c\% trading channel is defined as occurring when the high level of the price over the previous $j$ periods is within $\mathrm{c} \%$ of the low over the previous $j$ periods so that there are time-varying support and resistance levels that appear to be drifting together with about c\% or less separation. These time-varying support and resistance levels are 
the lower and upper bonds where the upper bound at a particular period will be $\mathrm{c} \%$ above the low of the previous $j$ periods and the lower bound will be $\mathrm{c} \%$ below the high of the previous $j$ periods. We examine two channel breakout rules, namely:

CB1: If a $c \%$ trading channel exists and if the price moves up at least $x$ percent above the upper bound of the channel and remains so for $d$ periods, go long. If a $c \%$ trading channel exists and if the price moves down at least $x$ percent below the lower bound of the channel and remains so for $d$ periods, go short.

CB2: CB1: If a $c \%$ trading channel exists and if the price moves up at least $x$ percent above the upper bound of the channel and remains so for $d$ periods, go long for $k$ periods and then neutralize the position. If a $c \%$ trading channel exists and if the price moves down at least $x$ percent below the lower bound of the channel and remains so for $d$ periods, go short for $k$ periods and then neutralize the position

We consider a number of different parameter specifications, namely:

$x \in\{0,0.01,0.05,0.1,0.5,1,5\}, \# x=7$

$d \in\{2,3,4,5\}, \# d=4$

$j \in\{2,5,10,25,50,75,100,150,200\}, \# j=9$

$c \in\{0.05,0.01,0.5,0.1,0.25,0.5\}, \# c=6$

$k \in\{3,5\}, \# k=2$

Therefore, we study the following number of parameterizations per rule:

$C B 1=\# x \times \# d \times \# j \times \# c=7 \times 4 \times 9 \times 6=1512$

$C B 2=\# x \times \# d \times \# j \times \# c \times \# k=7 \times 4 \times 9 \times 6 \times 2=3024$

Therefore the total $C B$ rules studied is 4536 .

The total number of different technical trading considered are: $7980+963+630+810$ $+4536=14,919$.

\section{References}

Allen, F., \& Karjalainen, R. (1999). Using genetic algorithms to find technical trading rules. Journal of Financial Economics, 51, 245-271.

Bajgrowicz, P., \& Scaillet, O. (2012). Technical trading revisited: False discoveries, persistence tests, and transaction costs. Journal of Financial Economics, 106, 473-491.

Bariviera, A. F. (2017). The inefficiency of bitcoin revisited: A dynamic approach. Economics Letters, 161, $1-4$.

Batten, J. A., Lucey, B. M., McGroarty, F., Peat, M., \& Urquhart, A. (2018). Does intraday technical trading have predictive power in precious metal markets? Journal of International Financial Markets, Institutions and Money, 52, 102-113.

Baur, D. G., Hong, K., \& Lee, A. D. (2018). Bitcoin: Medium of exchange or speculative assets? Journal of International Financial Markets, Institutions and Money, 54, 177-189.

Benjamini, Y., \& Hochberg, Y. (1995). Controlling the false discovery rate: A practical and powerful approach to multiple testing. Journal of the Royal Statistical Society, Series B, 57, 289-300.

Benjamini, Y., \& Yekutieli, D. (2001). The control of the false discovery rate in multiple testing under dependency. Annals of Statistics, 29, 1165-1188.

Bessembinder, H., \& Chan, K. (1998). Market efficiency and the returns to technical analysis. Financial Management, 27, 5-17.

Bouri, E., Molnár, P., Azzic, G., Roubaud, D., \& Lars Hagfors, I. H. (2017). On the hedge and safe haven properties of bitcoin: Is it really more than a diversifier? Finance Research Letters, 20, 192-198.

Brandvold, M., Molnár, P., Vagstad, K., \& Valstad, O. C. A. (2015). Price discovery on bitcoin exchanges. Journal of International Financial Markets, Institutions and Money, 36, 18-35.

Brauneis, A., \& Mestel, R. (2018). Price discovery of cryptocurrencies: Bitcoin and beyond. Economics Letters, $165,58-61$.

Brière, M., Oosterlinck, K., \& Szafarz, A. (2015). Virtual currency, tangible return: Portfolio diversification with bitcoin. Journal of Asset Management, 16, 365-373. 
Brock, W., Lakonishok, J., \& LeBaron, B. (1992). Simple technical trading rules and the stochastic properties of stock returns. Journal of Finance, 47, 1731-1764.

Cheah, E. T., \& Fry, J. (2015). Speculative bubbles in bitcoin markets? an empirical investigation into the fundamental value of bitcoin. Economics Letters, 130, 32-36.

Chordia, T., Goyal, A., \& Saretto, A. (2018). p-hacking. SSRN Available at: https://ssrn.com/ abstract=3017677 (pp. 32-36).

Ciaian, P., Rajcaniova, M., \& Kancs, D. (2018). Virtual relationships: Short- and long-run evidence from bitcoin and altcoin markets. Journal of International Financial Markets, Institutions and Money, 52, $173-195$.

Corbet, S., Lucey, B., Urquhart, A., \& Yarovaya, L. (2019). Cryptocurrencies as a financial asset: A systematic analysis. International Review of Financial Analysis, 62, 182-199.

Corbet, S., Lucey, B., \& Yarovaya, L. (2018). Datestamping the bitcoin and ethereum bubbles. Finance Research Letters, 26, 81-88.

Corbet, S., Meegan, A., Larkin, C., Lucey, B., \& Yarovaya, L. (2018). Exploring the dynamic relationships between cryptocurrencies and other financial assets. Economics Letters, 165, 28-34.

Detzel, A. L., Liu, H., Strauss, J., Zhou, G., \& Zhu, Y. (2018). Bitcoin: Learning, predictability and profitability via technical analysis. Available at SSRN: https://ssrn.com/abstract=3115846.

Dyhrberg, A. H. (2016a). Bitcoin, gold and the dollar-A GARCH volatility analysis. Finance Research Letters, 16, 85-92.

Dyhrberg, A. H. (2016b). Hedging capabilities of bitcoin. Is it the virtual gold? Finance Research Letters, 16, $139-144$.

Fry, J., \& Cheah, E. T. (2016). Negative bubbles and shocks in cryptocurrency markets. International Review of Financial Analysis, 47, 343-352.

Gencay, R. (1999). Linear, non-linear and essential foreign exchange rate prediction with simple technical trading rules. Journal of International Economics, 47, 91-107.

Han, Y., Hu, T., \& Yang, K. (2016). Are there exploitable trends in commodity futures prices? Journal of Banking and Finance, 70, 214-234.

Han, Y., Yang, K., \& Zhou, G. (2013). A new anomaly: The cross-sectional profitability of technical analysis. Journal of Financial and Quantitative Analysis, 48, 1433-1461.

Harvey, C., Liu, Y., \& Zhu, H. (2016).... and the cross-section of expected returns. Review of Financial Studies, 29, 5-68.

Harvey, C. R., \& Liu, Y. (2014). Evaluating trading strategies. The Journal of Portfolio Management, 40, $108-118$.

Hsu, P.-H., Taylor, M. P., \& Wang, Z. (2016). Technical trading: Is it still beating the foreign exchange market? Journal of International Economics, 102, 188-208.

Hudson, R., McGroarty, F., \& Urquhart, A. (2017). Sampling frequency and the performance of different types of technical trading rules. Finance Research Letters, 22, 136-139.

Kajtazi, A., \& Moro, A. (2019). The role of bitcoin in well diversified portfolios: A comparative global study. International Review of Financial, 61, 143-157.

Levich, R. M., \& Thomas, L. R. (1993). The significance of technical trading-rule profits in the foreign exchange market: A bootstrap approach. Journal of International Money and Finance, 12, 451-474.

Lintilhac, P. S., \& Tourin, A. (2017). Model-based pairs trading in the bitcoin markets. Quantitative Finance, 17, 703-716.

Marshall, B. R., Cahan, R. H., \& Cahan, J. M. (2008a). Can commodity futures be profitably traded with quantitative market timing strategies? Journal of Banking and Finance, 32, 1810-1819.

Marshall, B. R., Cahan, R. H., \& Cahan, J. M. (2008b). Does intraday technical analysis in the U.S. equity market have value? Journal of Empirical Finance, 15, 199-210.

Marshall, B. R., Nguyen, N. H., \& Visaltanachoti, N. (2017). Time series momentum and moving average trading rules. Quantitative Finance, 17, 405-421.

McLean, R. D., \& Pontiff, J. (2016). Does academic research destroy stock return predictability? The Journal of Finance, 71, 5-32.

Menkhoff, L. (2007). The obstinate passion of foreign exchange professionals: Technical analysis. European Journal of Finance, 145, 936-972.

Miffre, J., \& Rallis, G. (2007). Momentum strategies in commodity futures markets. Journal of Banking and Finance, 31, 1863-1886.

Moskowitz, T. J., Ooi, Y. H., \& Pedersen, L. H. (2012). Time series momentum. Journal of Financial Economics, 104, 228-250.

Nadarajah, S., \& Chu, J. (2017). On the inefficiency of bitcoin. Economics Letters, 150, 6-9.

Nakamoto, S. (2008). Bitcoin: A peer-to-peer electronic cash system. https://Bitcoin.org/Bitcoin.pdf. 
Narayan, P. K., Ahmed, H. A., \& Narayan, S. (2015). Do momentum-based trading strategies work in the commodity futures markets? Journal of Futures Markets, 35, 868-891.

Neely, C. J., Rapach, D. E., Tu, J., \& Zhou, G. (2014). Forecasting the equity risk premium: The role of technical indicators. Management Science, 60, 1772-1791.

Neely, C. J., Weller, P., \& Dittmar, R. (1997). Is technical analysis in the foreign exchange market profitable? A genetic programming approach. Journal of Financial and Quantitative Analysis, 32, 405-426.

Olson, D. (2004). Have trading rule profits in the currency markets declined over time? Journal of Banking and Finance, 28, 485-105.

Platanakis, E., Sutcliffe, C., \& Urquhart, A. (2018). Optimal vs naïve diversification in cryptocurrencies. Economics Letters, 171, 93-96.

Platanakis, E., \& Urquhart, A. (2019). Should investors include bitcoin in their portfolios? a portfolio theory approach. Available at SSRN: https://ssrn.com/abstract=3215321.

Politis, D., \& Romano, J. (1994). The stationary bootstrap. Journal of the American Statistical Association, 89, 1303-1313.

Poole, W. (1967). Speculative prices as random walks: An analysis of ten time series of flexible exchange rates. Southern Economic Journal, 33, 468-478.

Psaradellis, I., Laws, J., Pantelous, A. A., \& Sermpinis, G. (2019). Performance of technical trading rules: evidence from the crude oil market. European Journal of Finance, forthcoming.

Qi, M., \& Wu, Y. (2006). Technical trading-rule profitability, data snooping, and reality check: Evidence from the foreign exchange market. Journal of Money, Credit and Banking, 30, 2135-2158.

Shynkevich, A. (2012). Performance of technical analysis in growth and small cap segments of the us equity market. Journal of Banking and Finance, 36, 193-208.

Shynkevich, A. (2016). Predictability in bond returns using technical trading rules. Journal of Banking and Finance, 70, 55-69.

Smith, D. M., Wang, N., Wang, Y., \& Zychowicz, E. J. (2016). Sentiment and the effectiveness of technical analysis: Evidence from the hedge fund industry. Journal of Financial and Quantitative Analysis, 51, 1991-2013.

Sullivan, R., Timmermann, A., \& White, H. (1999). Data-snooping, technical trading rule performance, and the bootstrap. Journal of Finance, 354, 1647-1691.

Sweeney, R. J. (1986). Beating the foreign exchange market. Journal of Finance, 41, 163-182.

Szakmary, A. C., Shen, Q., \& Sharma, S. C. (2010). Trend-following trading strategies in commodity futures: A re-examination. Journal of Banking and Finance, 34, 409-426.

Taylor, M. P., \& Allen, H. (1992). The use of technical analysis in the foreign exchange market. Journal of International Money and Finance, 11, 304-314.

Timmermann, A., \& Granger, C. W. J. (2004). Efficient market theory and forecasting. International Journal of Forecasting, 50, 15-27.

Tiwari, A. K., Jana, R. K., Das, D., \& Roubaud, D. (2018). Informational efficiency of bitcoin-an extension. Economics Letters, 163, 106-109.

Urquhart, A. (2016). The inefficiency of Bitcoin. Economics Letters, 148, 80-82.

Urquhart, A. (2017). Price clustering in bitcoin. Economics Letters, 159, 145-148.

Urquhart, A., Gebka, B., \& Hudson, R. (2015). How exactly do markets adapt? Evidence from the moving average rule in three developed markets. Journal of International Financial Markets, Institutions and Money, 38, 127-147.

Urquhart, A., \& Zhang, H. (2019). Is bitcoin a hedge or safe haven for currencies? An intraday analysis. International Review of Financial, 64, 49-57.

White, H. (2000). A reality check for data snooping. Econometrica, 65, 1097-1126.

Yamamoto, R. (2012). Intraday technical analysis of individual stocks on the tokyo stock exchange. Journal of Banking and Finance, 36, 3033-3047.

Young, T. W. (1991). Calmar ratio: A smoother tool. Futures, 20(1), 40.

Young, T. W. (1991). Calmar ratio: A smoother tool. Futures, 20(1), 40.

Zarrabi, N., Snaith, S., \& Coakley, J. (2017). Fx technical trading rules can be profitable sometimes!. International Review of Financial Analysis, 49, 113-127.

Publisher's Note Springer Nature remains neutral with regard to jurisdictional claims in published maps and institutional affiliations. 